\section{Cahiers de Narratologie}

Analyse et théorie narratives

$18 \mid 2010$

Littérature et sciences

\title{
Du bon maniement de l'argent du roi en Espagne au XVIIIe siècle. Les enseignements d'un procès pour fraude
}

\section{Anne Dubet}

\section{(2) OpenEdition}

Journals

Édition électronique

URL : http://journals.openedition.org/narratologie/6080

DOI : $10.4000 /$ narratologie.6080

ISSN : 1765-307X

Éditeur

LIRCES

\section{Référence électronique}

Anne Dubet, « Du bon maniement de l'argent du roi en Espagne au XVIIIe siècle. Les enseignements d'un procès pour fraude », Cahiers de Narratologie [En ligne], 18| 2010, mis en ligne le 15 juillet 2010, consulté le 15 novembre 2019. URL : http://journals.openedition.org/narratologie/6080 ; DOI : $10.4000 /$ narratologie. 6080

Ce document a été généré automatiquement le 15 novembre 2019.

\section{(c) (i) (9)}

Cahiers de Narratologie - Analyse et théorie narratives est mis à disposition selon les termes de la licence Creative Commons Attribution - Pas d'Utilisation Commerciale - Pas de Modification 4.0 International. 


\section{Du bon maniement de l'argent du roi en Espagne au XVIIIe siècle. Les enseignements d'un procès pour fraude}

Anne Dubet

\section{NOTE DE L'AUTEUR}

*Les recherches que j'ai menées dans les archives ont été financées dans le cadre du projet ANR « Jeunes chercheurs et jeunes chercheuses » intitulé « Les grandes réformes de la comptabilité publique : racines, techniques, modèles » (projet 2006-2009).

Pour nombre d'historiens - dont je fais partie - les réformes financières entreprises par les premiers Bourbons d'Espagne au XVIII siècle n'affectent pas tant l'organisation fiscale de leurs territoires que leur administration. Avant que de modifier la répartition sociale des contributions, il s'agit de rendre au roi et à ses ministres de confiance la décision en matière de dépenses et de s'assurer de son exécution. Ce projet de contrôle accru du roi sur ses propres deniers se traduit certes par une modification en profondeur des relations établies avec les groupes d'intérêts privés, mais point par l'éviction de ces intérêts. Fermiers d'impôts, munitionnaires ou entrepreneurs deviennent des partenaires ordinaires, quand ils ne sont pas transformés en administrateurs, et l'on tend ainsi à régler leurs bénéfices'. On ne saurait donc confondre ce changement avec l'émergence de finances 
publiques au sens où les entendent les libéraux un siècle plus tard ${ }^{2}$. Afin d'en préciser l'ampleur et les limites, il est utile de se demander quelle est désormais la définition d'un bon administrateur et en quoi il se distingue de ses prédécesseurs du XVIIe siècle. Qu'est-il permis de faire avec l'argent du roi et qu'est-il souhaitable d'en faire?

2 Il est utile de passer par ce qui est interdit, la fraude, pour préciser ce qui est permis et souhaitable. Je m'attacherai ici à un cas ponctuel, le procès fait à Fernando Verdes Montenegro entre 1724 et 1728. Tour à tour trésorier général de la guerre (tesorero mayor), de 1721 à février 1724 , et Secrétaire d'État et des Dépêches chargé des Finances, jusqu'en octobre 1724, Verdes Montenegro est en butte dès le printemps de cette année-là à des accusations de fraude et d'erreurs multiples. Moins que la véracité des accusations, ce qui m'intéresse dans l'affaire est le discours des acteurs, le contenu qu'ils donnent aux concepts de fraude ou de bonne administration et l'usage qu'ils en font.

3 Ce sont les représentations des acteurs qui m'intéressent au premier chef. Il s'agit de comprendre quelles lignes de partage ils établissent entre la fraude et les comportements licites, mais aussi entre la bonne et la mauvaise administration en matière de finances royales, afin de mieux saisir quelle logique préside à la répartition du pouvoir et quelles fonctions ils assignent à la monarchie. L'interrogation est évidemment transposable aux sociétés contemporaines, et il est souhaitable de pouvoir disposer de suffisamment de monographies pour établir des comparaisons et cerner les continuités ou les ruptures dans le discours et les pratiques.

4 La thématique n'est pas neuve. L'étude de la corruption, ou ce que l'on appelle plus volontiers la fraude à l'époque moderne, a souvent été considérée comme un bon moyen d'accéder à la définition de la frontière entre sphères publiques et privées. L'ampleur du phénomène semble faire l'objet d'un certain consensus. En ce qui concerne les finances dans les territoires de la monarchie espagnole, on connaît bien diverses modalités de la fraude. Elles vont de l'évasion fiscale pratiquée par des contribuables plus ou moins modestes ${ }^{3}$ aux irrégularités commises par les plus puissants, qu'il s'agisse de favoriser des proches dans la répartition des impôts ou l'attribution de marchés municipaux ou royaux, de recevoir des pots-de-vin des heureux élus, de tirer un bénéfice personnel de l'endettement de sa ville ${ }^{4}$ ou de la connaissance qu'on a de 
l'état des caisses royales 5 . S'y ajoutent les abus de pouvoir commis par les percepteurs d'impôts à l'égard des contribuables ou par les responsables des caisses à l'égard des créanciers du roi ${ }^{6}$ et les vols et emprunts trop prolongés à la caisse dont on a la garde ${ }^{7}$. Les plus hauts personnages y participent parfois, tels ces vice-rois d'Amérique qui exigent des commissions des officiers nommés par le Conseil des Indes à Madrid pour les autoriser à occuper leur emploi ou s'enrichissent dans la contrebandes. Enfin, l'accent a été mis sur le peu d'efficacité de la répression en termes quantitatifs. Les pratiques frauduleuses ne cessent pas, puisque les textes de lois se répètent tandis que, lorsqu'il y a procès, il n'est pas rare que les accusés bénéficient de compositions réduisant leurs peines et restent en place' L'attitude de l'entourage royal paraît alors pragmatique - on transige faute de mieux, pour réduire les pertes ou éviter l'affrontement ${ }^{10}$-, quand la répression ne s'inscrit pas dans une stratégie politique (donner l'image d'un monarque vertueux, promouvoir une réforme) et financière ${ }^{11}$. Les finances de la France et d'états italiens du XVIe au XVIII ${ }^{\mathrm{e}}$ siècles ont suscité des observations similaires ${ }^{12}$.

5 Toutefois, au-delà du constat, les analyses divergent. Ainsi, Jean-Claude Waquet, prenant l'exemple de Florence ${ }^{13}$, renvoie dos à dos Van Klaveren, pour qui les pratiques corrompues seraient régies par la seule recherche du bénéfice économique, donc "amorales », et les héritiers de Max Weber, leur reprochant de croire que les acteurs des communautés qui ne participent pas de la modernité occidentale sont aussi pré-rationnels que leurs organisations politiques. Ceci conduit à son sens à des jugements de valeur douteux, contre lesquels nous préviennent aussi des anthropologues ${ }^{14}$ : tant les groupes demeurés en marge du processus de rationalisation en Europe que les habitants des pays africains victimes de la colonisation seraient arriérés, incapables de comprendre les nouvelles attentes des États centralisés, et donc innocents. Pour invalider ce schéma, Waquet rappelle que la corruption n'a pas disparu des formes d'organisation politique contemporaines ${ }^{15}$ et qu'à l'inverse, les acteurs de l'époque moderne ne sont pas ingénus. En effet, la corruption inquiète juristes et théologiens - il leur consacre un chapitre -, mais outre ces experts du légal et du licite, tous, et notamment les coupables et leurs juges, se sentent habilités à justifier ou condamner les pratiques soupçonnées de corruption au nom de principes moraux, 
d'usages ou de stratégies politiques. Waquet se propose donc de relire l'histoire des pratiques corrompues en prenant acte du fait qu'il s'agit bien de fautes pour ceux qui se défendent de les avoir commises comme pour leurs accusateurs. Suivant les présupposés de la sociologie fonctionnaliste, il s'agit d'en déceler la « fonction latente » dissimulée « derrière l'apparence de la dysfonction ». Plus que de compenser les petits salaires des juges et des employés aux finances ou de répondre à la demande des groupes exclus des jeux du pouvoir, la corruption, à son sens, permet de rétablir au profit du pouvoir un certain équilibre entre des élites plus ou moins bien implantées dans l'appareil d'État.

6 Cette lecture rend compte de l'importance de la fraude et des maigres résultats de la répression. En revanche, à mon sens, elle fait peu de cas de la culture des acteurs. En effet, Waquet considère a priori qu'il y a un décalage séculaire entre les pratiques des acteurs et leurs préceptes ${ }^{16}$ et paraît supposer que la vérité est dans les premières, que l'historien définit comme des fraudes même si ce n'en sont pas pour les acteurs. Il conçoit ainsi les distinguo établis par ces derniers comme un masque que l'on présente aux autres ou à soi-même pour atténuer la gravité des abus et continuer à les autoriser. Le Provéditeur Gondi, qui détaille dans son journal les cadeaux perçus pour ses intercessions auprès du Grand Duc de Venise, se ment en n'y voyant pas des pots-de-vin ${ }^{17}$. Les casuistes qui distinguent le cadeau spontané, fait sans arrière-pensée, de ce que l'on donne par calcul ou sous la menace et opposent le vol des deniers du souverain (péculat) à l'emprunt caché (reliquat) permettent aux corrompus de tout poil de frauder sans mauvaise conscience. Le probabilisme les autorise même à se donner pour règle non la plus probable (probabiliorisme), mais une des règles probables, fût-elle la plus laxiste ${ }^{18}$. La fraude est bien là, mais les acteurs veulent se le cacher ou le dissimuler aux autres et c'est à cette entreprise de dissimulation que paraissent se réduire les raisonnements les plus complexes. C'est donc bien la définition que l'historien donne à la fraude qui est valable. Une telle approche, si elle devait être radicalisée, autoriserait à faire l'économie de la lecture des sources dans lesquelles les acteurs expriment des jugements de valeur.

Parti d'un horizon théorique différent, Santos Madrazo parvient à des conclusions comparables. L'historien analyse la cause de Nicolás Ginés Gómez de Hinojosa, qui précéda 
puis succéda à Verdes Montenegro à la tête de la trésorerie générale en Espagne et fut accusé quelques années plus tard de péculat. D'après lui, ce cas n'est qu'un des exemples des «vols» qu'implique une «structure de pouvoir de classe de cette société19 ». En effet, les classes dominantes s'accordent pour faire un usage privé de l'État - même si l'auteur refuse d'aborder la définition des sphères publique et privée -, comme l'indique le titre du livre, État faible et voleurs puissants. La norme voudrait que ces vols soient impunis, voire récompensés : seules les rivalités entre clientèles conduisent parfois à des procès, les peines étant alors atténuées après tractations. Des juristes et théologiens étant solidaires des classes dominantes, ni le droit ni la morale ne sauraient favoriser une répression rigoureuse. Le premier définit plus clairement les vols commis par les plus faibles que le péculat, à peine mentionné dans certains traités, quoique passible de lourdes peines. La seconde, voyant dans le péculat un péché, soit une faute individuelle, nie le contenu social et politique des pratiques corrompues et offre une casuistique avantageuse à leurs adeptes. De fait, une culture religieuse dans laquelle «les relations avec Dieu passent par des intermédiaires spirituels et temporels qui admettent ce type de pratiques » s'accommode forcément de la corruption ${ }^{20}$ ! Enfin, l'État, machine à réprimer les classes inférieures et à extraire l'excédent produit par les paysans - l'argent volé vient de là -, est mu par la «volonté politique d'obtenir que les gens soient socialement indifférents devant les pratiques de malversation et de corruption ${ }^{21}$. Cette critique de la domination, que Madrazo étend à l'actualité ${ }^{22}$, éveille la sympathie. Cependant, la démonstration repose sur ce postulat indémontrable que seul l'historien a le privilège de l'extériorité et de la lucidité, quand les acteurs, bénéficiaires ou non de la domination, partagent l'illusion que la fraude n'est qu'un délit ou un péché dont on peut s'arranger avec juges et confesseurs. Il est alors presque anecdotique que, dans les accusations portées contre le Trésorier Général, le mot « vol » ne soit pas prononcé et que l'on ne parle pas de son péculat en termes péjoratifs - de fait, on exige seulement qu'il rende raison d'un "solde de compte favorable aux finances du roi » et « autres périphrases melliflues ${ }^{23}$ ».

Quel que soit le jugement de valeur que l'on porte sur les interprétations des acteurs que l'on étudie - même s'ils se trompent -, j'estime prudent de ne pas s'en débarrasser si l'on veut comprendre les raisons de leurs actions. Dans le 
cas présent, il n'est pas illogique d'admettre qu'ils ne sont ni amoraux ni candides tout en s'efforçant de reconstruire des catégories morales qui ne sont plus les nôtres. Cette position, que l'on pourrait rattacher à celle des anthropologues ou de certains wébériens, passe par une lecture plus attentive aux nuances qu'ils établissent entre le licite et l'illicite, que ces nuances soient ou non des prétextes.

9 Ainsi, il convient de souligner que la différence nette établie par les juristes et les théologiens analysés par Waquet entre des cadeaux offerts par pure libéralité ou gratitude, et sans calcul, et ceux qui sont imposés par officiers et magistrats (concussion) ou qui leur sont offerts dans l'intention d'en obtenir une contrepartie (corruption), n'est pas un raffinement d'experts. Le même historien, on l'a vu, cite des officiers et des juges qui souscrivent aux mêmes lignes de partage. Il n'est pas le seul : les recherches de Mireille Peytavin sur les visites générales menées pendant deux siècles dans différents territoires de la monarchie espagnole ont mis au jour des discours similaires ${ }^{24}$. Il conviendrait de multiplier les témoignages et de vérifier qu'en dehors des élites habituellement impliquées dans la corruption des finances royales, d'autres secteurs de la société partagent ces arguments. Mais l'on peut d'ores et déjà supposer que cette différence entre ce qui est libre et ce qui ne l'est pas, si elle est partagée par toutes les élites d'une société d'Ancien Régime, n'est pas anecdotique. Elle est abondamment glosée par les contemporains dans d'autres domaines, comme la fiscalité (un service n'est pas un impôt), les obligations du roi à l'égard de ses sujets (la grâce s'oppose au paiement dit de justice ${ }^{25}$ ), le prêt d'argent (il est interdit de payer l'acte de prêter, mais pas de faire un cadeau par gratitude ${ }^{26}$ ) ou la justice (on peut appliquer le droit pénal, mais aussi pardonner ${ }^{27}$ ). Pourquoi ne pas la prendre au sérieux dans celui de la fraude, à l'instar des partisans de l'anthropologie historique ${ }^{28}$ ? Cela ne facilite certes pas la mesure de certaines formes de fraude. S'il ne peut y avoir corruption sans intention de corrompre ou d'être corrompu, savoir qu'un justiciable ou un contribuable a donné quelque chose à un officier ne suffit pas plus à l'historien qu'au juge. Mais observer que le débat entre les acteurs a porté sur la nature du don et non sur son existence et qu'un cadeau purement gracieux en théorie n'était pas jugé répréhensible offre des indices précieux sur la culture politique moderne. 
10 En ce qui concerne les actes commis au sein de l'administration des finances par ceux qui ont accès aux deniers du souverain, Waquet rappelle que les contemporains distinguent le péculat du reliquat. Le premier consiste à s'approprier les deniers du souverain. Le second est une rétention provisoire, son auteur ayant l'intention de rendre l'argent qu'il a pris dans la caisse sans le dire une fois qu'il en aura tiré bénéfice. Waquet indique que juristes et théologiens sont plus cléments à l'égard du reliquat: le droit invite les juges à arbitrer, plusieurs théologiens précisent qu'il n'y a pas faute dans la mesure où l'argent est restitué au moment où cela est nécessaire ${ }^{29}$. De fait, le terme de reliquat ne désigne pas toujours un délit. Le Dictionnaire des finances (1727) le définit de façon neutre : est reliquataire « le comptable qui doit une somme par l'arrêté de son compte ». Pour qu'il y ait faute, il faut d'abord que le reliquat soit le résultat d'une rétention, c'est-à-dire que le comptable ait soustrait de l'argent à la caisse sans le dire, ce qui suppose souvent des manipulations comptables : on omet ou retarde l'enregistrement de recettes effectives, commettant alors omission et occultation, ou, plus rarement, l'on invente des dépenses qui n'ont pas eu lieu ou ont eu lieu plus tard. Ces pratiques, notamment la première, sont monnaie courante, si l'on en croit les règlements pour la tenue des livres ou les questionnaires des enquêtes dont peuvent faire l'objet les comptables. Or, il semble facile de les dissimuler, pour peu que le comptable s'accorde avec le contrôleur chargé de tenir les livres de comptes de sa caisse, ou avec des créanciers qui acceptent d'être payés plus tard si on leur verse un intérêt ou qui n'ont pas le choix ${ }^{30}$. Le mode de tenue des livres en vigueur dans les finances royales, municipales, et dans toute administration de biens confiée à un tiers, n'est pas fait pour rendre visible la rétention au moment où elle se produit, puisque les comptes ne sont rendus qu'après la fin de l'exercice, reflétant seulement la position du comptable par rapport à son mandataire à ce moment-là. Un emprunt dissimulé et remboursé avant la fin de l'exercice peut donc passer inaperçu, s'il n'a pas conduit le comptable à suspendre des paiements impératifs ${ }^{31}$. Or, le choix de ce mode de tenue des livres est assumé : pour preuve, l'introduction de la partie double, qui rendrait plus difficile l'occultation, rencontre l'hostilité ${ }^{32}$. Il importe donc de déterminer si toute rétention est coupable, ou ce qui la rend coupable, pour comprendre la nature de la fraude. Or, certains financiers, à l'instar des spécialistes cités par 
Waquet, distinguent des rétentions coupables d'autres qui sont tolérables: c'est le cas quand ils affirment que le monarque ne saurait attirer de financiers crédibles (pour tenir ses caisses ou servir de cautions à ceux qui les tiennent) que s'il leur laisse la possibilité de faire du profit avec ses deniers - donc, de pratiquer la rétention -; en effet, les salaires médiocres qui leur sont versés ne sauraient séduire que des gens peu fiables ${ }^{33}$. C'est pourquoi plus que le reliquat ou rétention en soi, il semble que ce sont ses conséquences qui peuvent en faire un acte coupable. Il est souhaitable de trouver des indices supplémentaires du fait que cette opinion est partagée par les contemporains. En effet, cela viendrait confirmer qu'il est licite de faire du profit avec les deniers du roi, tant que le roi n'a pas à en supporter de conséquences dommageables, et en retire par ailleurs le bénéfice de pouvoir confier son argent à des gens de crédit. Je crois que Santos Madrazo ne tient pas compte de cette nuance parce qu'il conçoit l'argent du roi catholique comme des « fonds publics ».

11 L'affaire Verdes Montenegro donne accès, dans un cas précis, à l'interprétation et à l'usage que font des acteurs du concept de fraude. En effet, les volumineux dossiers conservés $^{34}$ comportent notamment les textes des accusations et de la défense, qui exposent les motifs de la culpabilité ou de l'innocence du ministre et, par là, donnent un contenu au concept. L'affaire présente un intérêt supplémentaire. Il est mis en cause comme trésorier qu'il fut et comme ministre des finances. Dans son second emploi, Verdes Montenegro n'est pas seulement accusé de malversation de fonds, mais aussi d'incompétence. Son cas pourrait ainsi illustrer des attentes nouvelles à l'égard des administrateurs des deniers du roi.

12 En février 1724, Fernando Verdes Montenegro, Trésorier Général de la caisse de la guerre, devient Secrétaire d'État et des Dépêches chargé des Finances, succédant au marquis de Campoflorido, qui vient de renoncer à son emploi après l'abdication de Philippe $\mathrm{V}$, alléguant sa mauvaise santé. Nicolás Ginés Gómez de Hinojosa prend la direction de la caisse à compter du $1^{\text {er }}$ mars suivant ${ }^{35}$. Tous trois sont des financiers expérimentés. Le plus âgé, Campoflorido, a été Trésorier Général des dépenses de la guerre (Tesorero Mayor) pendant la Guerre de Succession et a eu plusieurs fois des fonctions de surintendant des finances; depuis 1720, outre le Secrétariat, il avait le 
Gouvernement du Conseil des Finances et la surintendance des rentes générales et de la recette du tabac. Hinojosa a été Trésorier Général des dépenses de la guerre (Tesorero Mayor) puis Trésorier Général (Tesorero General) entre 1717 et 1720 , et Verdes lui a succédé le $1^{\mathrm{er}}$ janvier 1721, comme Trésorier Général des dépenses de la guerre ${ }^{36}$. A ce titre, ils ont été associés à la consolidation de deux organismes solidaires, mis en place au cours de la Guerre de Succession pour donner à l'administration des finances un tour plus " exécutif», une caisse seule habilitée à faire les dépenses de la guerre, placée sous l'autorité directe d'un Secrétaire des Dépêches pensé comme ministre responsable, qui ne rend de comptes qu'au roi ${ }^{37}$. Or, c'est aussi des compétences respectives du Trésorier et du Secrétaire aux Finances qu'il est question dans l'affaire Verdes Montenegro.

13 Le déroulement de la cause est retracé par Santos $\mathrm{Madrazo}^{38}$. L'auteur en démontre la dimension politique, confirmée par les acteurs. A l'origine du conflit, le dépit de Campoflorido de voir Verdes Montenegro occuper le Secrétariat des Dépêches aux Finances et la surintendance auxquelles il a renoncé à l'avènement de Louis Ier, tandis que le gouvernement du Conseil des Finances est confié à don Juan Blasco de Orozco. Verdes Montenegro bénéficie alors de la protection du gouverneur de Castille, le marquis de Miraval, qui a gagné en influence ${ }^{39}$ et le recommande pour ces emplois ${ }^{40}$. Peu de temps après, Verdes Montenegro est en désaccord ouvert avec Hinojosa, qui n'accepte pas les modifications que le nouveau ministre prétend apporter aux règles régissant sa trésorerie. C'est Hinojosa qui obtient gain de cause en mars, grâce à l'appui de don Juan Bautista de Orendain, Secrétaire des Dépêches et d'État, le roi obligeant Verdes Montenegro à réformer son texte ${ }^{41}$. Autre source de conflit en mai 1724, le choix des intermédiaires pour les lettres de change pour l'Espagne péninsulaire et pour l'extérieur, Verdes Montenegro refusant ceux proposés par Hinojosa, au motif que leurs tarifs sont supérieurs à ceux qu'il a pu trouver ${ }^{42}$. Le plan de dotation des dépenses soumis par le ministre au roi en mai est attaqué par Campoflorido (15 juillet) puis Hinojosa (3 août), qui lui reprochent d'avoir oublié d'y inclure des restes des recettes de 1723 et engagent un bras de fer pour le convaincre de faire un emprunt pour les paiements les plus urgents. La rapidité avec laquelle le second produit des documents quand le roi l'interroge sur l'avis émis par le 
premier pourrait confirmer qu'ils se sont concertés, et c'est là ce que croient Verdes ${ }^{43}$ et, plus tard, la junte qui juge l'affaire. Après la mort précoce de Louis Ier, en août, et le retour à Madrid de ministres hostiles à Miraval, d'autres accusations sont portées contre Verdes, qui conduisent Philippe V à le démettre de ses emplois (27 octobre 1724) et à l'exiler pour permettre à l'enquête de se dérouler. Celle-ci, menée par une junte à partir de l'été 1725, se conclut par la levée de la réhabilitation et la restitution à l'intéressé des honneurs et émoluments perdus (1728-1738). C'est à ce moment-là qu'Hinojosa fait l'objet de premiers soupçons ${ }^{44}$, et il est tentant d'y voir une contreattaque des amis de Verdes Montenegro.

14 Cela dit, l'affrontement entre clientèles n'épuise pas l'affaire. Les arguments mobilisés contre l'accusé ou pour assurer sa défense ont à l'évidence une valeur stratégique, et de fait, l'auteur des conclusions de la junte, en 1727, a beau jeu de signaler que ceux-là mêmes qui accusent Verdes Montenegro d'avoir diverti des recettes avant l'été 1724 les font apparaître au lendemain de son éviction ${ }^{45}$. Toutefois, même si les accusations obéissent à un calcul, on peut faire l'hypothèse que ceux qui les avancent les jugent assez crédibles pour être présentées au roi. Ainsi, s'ils ne croient peut-être pas en leur for intérieur que Verdes Montenegro a commis ce dont on l'accuse, ils sont probablement persuadés que ce dont on l'accuse est condamnable et sera jugé tel par tous. C'est en cela que leur discours permet de dessiner le portrait idéal d'un mauvais trésorier et d'un mauvais ministre.

De quoi dit-on Verdes Montenegro coupable ? Le procureur du Conseil des Finances résume les accusations à quatre points en février $1726^{46}$ : 1 / dans un état prévisionnel des dépenses et recettes de l'année 1724 présenté au roi en février 1724, Verdes Montenegro a omis d'inclure des fonds considérables, restes (residuos) des recettes de 1723, alors qu'il y a porté des dettes héritées de 1723 ; 2/ il a en outre affirmé dans cet état qu'il n'y avait pas d'autres capitaux pour augmenter les fonds de l'année ; 3 / après la fin de son exercice de Trésorier Général des dépenses de la guerre, il a continué d'émettre des quittances pour encaisser des recettes; 4/ enfin, connaissant l'indigence de nombreux créanciers du roi, il leur a préféré le munitionnaire des vivres de Catalogne.

16 Ces accusations mêlent divers arguments. La plus récurrente est l'accusation d'occultation, terme employé 
par Campoflorido et Hinojosa dès l'été 1724. Elle s'accompagne d'un soupçon de mauvais usage de l'argent : un résumé de l'affaire produit par la junte le 15 juin 1727 évoque « ladite occultation, malversation et divertissement de fonds ${ }^{47} \gg$. De fait, il semble que l'occultation ne suffise pas pour convaincre le ministre de culpabilité. C'est ce qu'il a fait de l'argent non déclaré dans l'état prévisionnel de février 1724 qui pourrait le rendre coupable. Une première junte, réunie en octobre 1724 pour examiner l'opportunité de prononcer une accusation formelle, hésite entre plusieurs possibilités; soit il a agi :

dans l'intention de se rendre indispensable dans le ministère en sous-évaluant les fonds et en grossissant les dépenses pour pouvoir parvenir, avec les fonds qu'on n'inscrivait pas [dans l'état prévisionnel], à se tirer sans grande peine brillamment de la difficulté, soit il l'a fait dans l'intention de les utiliser dans quelques négociations, ce pour quoi il y a quelques présomptions. ${ }^{48}$

17 Les auteurs précisent: «quoique la malversation de capitaux et les négociations illicites faites avec ne soit pas pleinement vérifiée, il y a pour le moins nombre d'indices, conjectures et présomptions qu'il y en a eu », à quoi s'ajoute « la rumeur qui veut qu'on ait négocié les billets »49. L'hypothèse du calcul politique semble ne pas avoir été retenue, car on n'en trouve pas trace dans les défenses écrites de Verdes Montenegro. En revanche, le soupçon de bénéfices personnels demeure. C'est dans le cadre de ses fonctions de trésorier qu'il se serait livré à un fructueux trafic, secondé par une dizaine de complices : il s'agissait de « recueillir des quittances en sa faveur », en ne payant pas entièrement les assignations reçues et en donnant aux créanciers des billets; lorsque ceux-ci les revendaient au rabais, des complices de Verdes Montenegro les rachetaient pour les faire valoir dans la trésorerie ${ }^{50}$.

Un autre soupçon de profit illicite est enfin apporté au dossier. Le 4 novembre 1724, le maréchal de Tessé promet à Orendain de l'informer sur le paiement de 25000 doublons à l'Électeur de Bavière, une dette qui court depuis 1717, mais qui aurait dû passer après des dettes plus urgentes. Au fil de l'enquête menée sur cette opération en 1725 et 1726, l'accusation se précise : le confesseur de la reine abrite "un soupçon fondé de subornation et de collusion », puisqu'à lui aussi on a tenté d'offrir 50 doublons pour qu'il obtienne que le paiement soit effectué. Il ajoute plus tard qu'il « convient beaucoup au service du roi d'interdire aux ministres de payer des arriérés sans ordre exprès de SM, afin d'éviter des transactions que seul le roi est en droit de faire ${ }^{51} »$. Verdes Montenegro aurait 
ainsi pu se laisser corrompre pour accepter de faire passer un créancier secondaire avant les autres.

Mais ce n'est pas seulement le soupçon d'enrichissement illicite qui pèse sur Verdes Montenegro. Les accusations concernent aussi ses compétences de ministre. Tout d'abord, il aurait dû rendre raison au roi de l'emploi qu'il faisait de tous les fonds. Lorsque Campoflorido l'accuse d'avoir occulté 4,5 millions de recettes dans l'état de février 1724 (les restes de 1723), et, après la fin de son mandat de trésorier, d'avoir continué à faire rentrer l'argent, c'est pour le « distribuer selon son gré en l'employant à d'autres fins $^{52}{ }^{2}$. En outre, en tant que ministre des finances, Verdes Montenegro a mal choisi les dépenses à privilégier. Il est en effet responsable du fait que les maisons royales, le personnel des Conseils et des bureaux des Secrétaires des Dépêches à Madrid n'ont pas été payés. D'après la défense, les ennemis de Verdes Montenegro vont alors jusqu'à écrire six fausses lettres prouvant que les troupes ont été laissées à l'abandon, de sorte qu'en août 1724, «tout périssait », ce qui provoqua la «consternation » de Louis Ier qui mourut peu après ${ }^{53}$.

20 Faute supplémentaire énoncée par Hinojosa, Verdes Montenegro aurait dû connaître les usages propres au financement des monarchies. En effet, alors que le Trésorier prône un emprunt remboursable en quatre mois sur l'argent des Indes attendu en 1725, qui permettrait d'attendre à peu de frais que les recettes fiscales rentrent, le ministre prétend ne compter que sur les recettes de 1724 pour assurer les dépenses de l'année : cela l'obligera à recourir à des avances sur recettes dans certaines provinces et pas dans d'autres, contre toute équité ; dans la pratique, cela offrira aux "subalternes » des provinces une marge de manœuvre illégitime, puisque, privés de fonds suffisants pour assurer les dépenses qu'ils sont tenus de faire, ils agiront selon leurs "préférences indues ». Le jugement est sans appel : « Reste à expliquer comment, ces règles n'ayant rien de métaphysique, on propose des moyens contraires, car il n'est pas facile de concéder que tout praticien moyen puisse les ignorer ${ }^{54}$ ». En somme, « la Providence n'accorde pas tout à tout le monde ${ }^{55} \gg$. Verdes Montenegro a bien compris l'allusion, comme l'indique sa défense : Hinojosa «qualifi[ait] de bêtise le fait de vouloir satisfaire aux obligations de la monarchie avec les fonds de l'année ${ }^{56} »$. Enfin, c'est encore la compétence du ministre qui est en cause lorsque Campoflorido lui reproche de ne 
pas avoir su ce qu'il fallait faire figurer ou pas dans l'état prévisionnel de février 1724 et de ne pas avoir fait en sorte d'obtenir l'information qui lui manquait pour les préparer correctement, alors que c'était son devoir. L'accusation, reprise par le procureur, est ainsi résumée dans la défense de Verdes Montenegro : il est accusé « pour avoir accepté l'honneur que le roi a bien voulu lui faire, sans savoir le métier qu'il lui confiait ${ }^{57}{ }^{\prime}$.

21 On s'en doute, Verdes Montenegro se dit aussi innocent devant Dieu que saint François. Pour le prouver devant le tribunal du roi, il répond point par point aux accusations. En tant que Trésorier Général de la caisse des dépenses de guerre, il n'a pu faire rentrer de recettes sans le dire après la fin de son mandat. L'argument technique renvoie au mode de contrôle des comptes. Comme ses prédécesseurs dans la Trésorerie, il n'était plus autorisé à émettre de quittances pour les rentrées de fonds. S'il avait enfreint l'interdit, ses quittances n'auraient pas été valables puisque non visées par le contrôleur de la Trésorerie. Il n'aurait donc pu commettre ce délit sans que coopèrent

les contrôleurs, ceux qui auraient remis les fonds, ceux qui les auraient reçus, et finalement, tant de gens, que l'on ne pourrait le cacher, et ainsi la chose était impraticable, même si j'avais été un homme sans religion et sans foi, et même ainsi, j'aurais toujours eu l'obligation de rendre compte, de sorte que cela n'aurait servi à rien $^{58}$.

Verdes Montenegro rappelle par ailleurs que c'est bien lui qui a tout fait à la fin du mois de février 1724 pour rendre plus étroit le contrôle exercé sur la Trésorerie. Les modifications qu'il avait introduites dans le règlement visaient en effet à réduire son autonomie : le texte affirmait que le Trésorier était subordonné au seul Secrétaire des Dépêches et d'État aux Finances, qu'il ne pouvait payer les dettes que lui transmettait son prédécesseur sans ordre exprès du roi, transmis par ledit ministre, et enfin il instituait un contrôle nouveau sur la caisse de la Trésorerie. C'étaient ces clauses qu'Hinojosa avait fait annuler en mars $^{59}$. Quant à subtiliser les 4,5 millions d'écus en tant que ministre, c'était tout aussi impossible, puisque « le ministre des finances ne reçoit pas d'argent et ne peut en user, car tout doit aller obligatoirement au Trésorier Général ${ }^{60}$ ». Une minutieuse démonstration examine, partie par partie, le compte dressé par Campoflorido et celui d'Hinojosa. Elle établit que chacune des sommes qu'ils présentent comme des restes de recettes de 1723 sont soit des sommes dont la dépense était déjà engagée en 1723 - à ce titre, elles n'ont pas à figurer dans l'état de février 1724 -, soit, dans le cas de vivres restés en magasins, des biens que l'on ne compte 
jamais comme un fonds à dépenser - car on les conserve comme réserves pour les situations d'urgence. Les sommes inscrites en blanc dans le même état le sont ainsi tous les ans car on n'en sait pas le montant en début d'année. Enfin, des comptables de recettes ont avoué avoir menti pour servir les desseins de Campoflorido. À l'appui de ce texte, des relations réclamées aux contrôleurs de chacune des recettes portées dans l'état de 1724, et plus tard, un exposé des contrôleurs de la Trésorerie Générale pour les dépenses de guerre qui indique comment sont établis les états prévisionnels en début d'année - cet exposé confirme le principe de l'annualité des états de fonds et indique que, n'étant pas des redditions de compte, ils ne peuvent servir de base à une accusation formelle. C'est donc le marquis qui a voulu mal lire les relations sur lesquelles il s'est appuyé pour constituer son propre état et qui a oublié l'annualité. La conclusion de Verdes Montenegro est double: d'une part, le soupçon d'occultation tombe, puisqu'il n'y avait rien à occulter, et avec lui les éventuelles "négociations illicites » et les «malversations »; d'autre part, l'accusé maîtrise mieux les documents comptables que Campoflorido.

23 C'est bien sur son talent de ministre des Finances, et auparavant, de Trésorier, qu'il insiste. Sa capacité à dresser des états de dépenses et recettes ne date pas de 1724 . Il rappelle en effet que c'est lui qui, lorsqu'il était Trésorier, a préparé ceux présentés au roi par Campoflorido, avec l'assentiment de tous les praticiens. Mais sa virtuosité est plus grande encore, car il n'a pas besoin des papiers, maîtrisant parfaitement les dossiers. Lors d'une junte réunie peu avant la mort de Louis Ier pour examiner l'état prévisionnel qu'Hinojosa opposait au sien, il a ainsi fait taire ses ennemis :
et les ministres qui y ont participé ont vu comment, après qu'Hinojosa avait passé beaucoup de temps à l'établir, s'appuyant sur celui que j'avais fait et lui avais donné avec tous les papiers et matériaux qu'il avait et garde en son pouvoir, et qu'il s'était réuni avec le marquis de Castelar pour le mettre au point, il a apporté son œuvre à la junte, et moi, sans en avoir vu une lettre ni rien savoir de son contenu, je lui ai posé de mémoire et sans prendre la plume, en présence de tous, les questions suffisantes pour vérifier et montrer la sûreté de mon propre état, dans des termes tels que le marquis de Castelar a interrompu la réunion, insistant sur le fait qu'il n'y avait aucune objection [à mon état] et que celui d'Hinojosa n'y ajoutait rien. Hinojosa l'a reconnu et l'on est ainsi passé à d'autres matières ${ }^{61}$.

Verdes Montenegro offre d'ailleurs à Grimaldo de reproduire l'exploit en public, si ses ennemis acceptent le défi62. C'est cette maîtrise des dossiers acquise en tant que Trésorier qu'a mise en avant Miraval pour le recommander 
comme ministre : " une connaissance parfaite de toutes les troupes, dans toutes leurs parties, chose si difficile à comprendre pour qui n'a pas eu de pratique dans ce maniement ${ }^{63} \gg$. Mais ce portrait n'est pas que celui d'un bon technicien. Verdes Montenegro revendique son discernement dans les choix faits pour les dépenses. Ainsi, en Catalogne, il a payé le prest et non la provision des vivres, mais l'un est aussi urgent que l'autre. S'il faut incriminer quelqu'un pour avoir mal organisé les dépenses, c'est Campoflorido, ministre qui n'a laissé que des dettes ${ }^{4}$. Quant à Hinojosa, Verdes Montenegro l'accuse formellement dès juin de suspendre le paiement du personnel des ministères pour allonger la liste de ses ennemis, alors que les recettes destinées à cet emploi sont disponibles et que, dans les provinces, les intendants font les dépenses ordonnées ${ }^{65}$.

Pour sa part, Verdes Montenegro se dépeint comme un modèle de vertu financière. Sa défense insiste sur le fait qu'il a su assurer les dépenses de 1724 avec les seules recettes de l'année et, lorsqu'il fallait recourir à des financiers privés, trouver les plus fiables et les moins chers : c'est le cas pour les changeurs avec qui il voulait passer contrat pour la Péninsule et l'extérieur - Hinojosa l'en a empêché - ainsi que pour le financier Urban Velarde, dont il a pu obtenir une avance pour le paiement du prest de Catalogne bien moins coûteuse que les prix que l'on acceptait (c'est-à-dire, que Campoflorido acceptait) les années précédentes ${ }^{66}$. Pour finir, avant 1724, Verdes Montenegro croit avoir été un trésorier bien supérieur à Hinojosa. En effet, déjà, alors qu'il était trésorier d'armée de Catalogne sous le comte de Moriana, il a pu soutenir les troupes par son crédit,

quand il en était trésorier [de l'armée], les troupes de Sa Majesté Très Chrétienne étant retenues à Barcelone en 1714 après la reprise de la place, et alors que les fonds manquaient pour les payer, ils se sont contentés pour tout ce qui leur était dû de billets de don Fernando, demandés par le maréchal de Berwick et le chevalier d'Asfeldt, à qui il les a remis ${ }^{67}$.

$\mathrm{Au}$ contraire, pendant les exercices successifs d'Hinojosa comme Trésorier Général (des dépenses de guerre ou tout court), les troupes envoyées en Sicile n'ont guère été payées :

car de l'année 1716, où il l'était devenu [Trésorier Général] pour la première fois jusqu'à la fin de l'année 1720, les mêmes comptables indiquaient qu'on avait tant manqué de fonds que l'on avait presque cessé d'assister les troupes, comme le montrait le fait qu'étant restées 28 mois en Sicile, celles qui avaient participé à l'expédition dans ce royaume n'avaient reçu que 7 mensualités ${ }^{68}$. 

qualités exigées des financiers $\mathrm{du}$ roi. Un bon administrateur des deniers du roi ne peut se contenter d'être vertueux. Il faut qu'il ait une culture financière qui passe par la maîtrise des techniques de comptabilité et d'établissement de budgets prévisionnels, mais aussi la connaissance des pratiques de crédit propres aux monarchies. Si le Trésorier, simple exécutant, n'est pas supposé aller au-delà, il se trouve que, dans le cas présent, c'est le Trésorier qui prétend donner des leçons au ministre, qui le lui rend bien. En octobre 1726, José Patiño, tout puissant responsable des Finances, clarifiera la répartition des rôles. Quant au ministre, il est supposé ajouter à ces talents un sens aigu de l'opportunité, sachant qui payer et qui faire attendre, puisque le roi ne peut satisfaire tout le monde. Enfin, si l'on en croit Verdes Montenegro - mais c'est là qu'il échoue - il doit pouvoir imposer sa politique aux exécutants.

31 Cette revendication d'un savoir et d'un savoir-faire propre aux bons administrateurs, dont on peut être fier et qu'on peut opposer à ceux qui ne sont pas gâtés par la Providence, n'est pas isolée au début du XVIII ${ }^{\mathrm{e}}$ siècle $^{71}$. Il est 


\section{tentant d'y voir un indice d'une profonde transformation de l'administration des finances.}

\section{BIBLIOGRAPHIE}

Alonso García (David) : 2007b, El erario del reino. Fiscalidad en Castilla a principios de la Edad Moderna (1504-1525), Valladolid, Junta de Castilla y León.

Alonso García (David) : 2008, « Finances royales et monde financier dans la création de la monarchie espagnole (XVI ${ }^{\mathrm{e}}$ siècle) » dans Dubet éd.: 2008, p. 175-186.

Andrés Ucendo (José Ignacio) : 1999, La fiscalidad en Castilla en el siglo XVII : los servicios de millones, 1601-1700, Universidad del País Vasco.

Bernardo Ares (José Manuel de) : 1993, Corrupción política y centralización administrativa. La hacienda de propios en la Córdoba de Carlos II, Cordoue, Universidad de Córdoba.

Bertrand (Michel) : 1999, Grandeur et misère de l'office de Nouvelle-Espagne (XVII ${ }^{e}$-XVIII ${ }^{e}$ siècles), Paris, Publications de la Sorbonne.

Bertrand (Michel) : 2008, « Administrer les finances aux Indes : entre service royal et intérêts familiaux (XVII ${ }^{\mathrm{e}}$-XVIII ${ }^{\mathrm{e}}$ siècles) » dans Dubet éd. : 2008, p. 213-226.

Cárceles de Gea (Beatriz) : 1995, Reforma y fraude fiscal en el reinado de Carlos II. La Sala de Millones (1658-1700), Madrid, Banco de España.

Cárceles de Gea (Beatriz) : 2000, Fraude y desobediencia fiscal en la corona de Castilla (1621-1700), Junta de Castilla y León.

Carlos Morales (Carlos Javier de), 2000, Carlos V y el crédito de Castilla. El tesorero general de Francisco de Vargas y la Hacienda Real entre 1516 y 1524, Madrid, Sociedad Estatal para la Conmemoración de los Centenarios de Felipe II y Carlos V.

Castro (Concepción de) : 2004, A la sombra de Felipe V. José de Grimaldo, ministro responsable (1703-1726), Madrid, Marcial Pons Historia.

Clavero (Bartolomé) : 1986, Tantas personas como estados. Por una antropología política de la historia europea, Tecnos.

Clavero (Bartolomé) : 1991, Antidora. Antropología católica de la economía moderna, Milan, Giuffrè Editore.

Clavero (Bartolomé) : 1996, « La monarquía, el derecho y la justicia », E. Martínez Ruiz, M. De Pazzis Pi et al., Instituciones de la España moderna. 1. Las jurisdicciones, Madrid, Actas Editorial, p. 15-38.

Cuartas Rivero (Margarita) : 1984, «El control de los funcionarios públicos a finales del siglo XVI », Hacienda Pública Española, n 87, p. 145-173.

Dessert (Daniel) : 1984, Argent, pouvoir et société au Grand Siècle, Paris, Fayard.

Díaz Ordóñez (Manuel) : 2003, « Lo legal y lo ilegal en la contratación del asiento de jarcía para la armada española », Tiempos modernos (Revista electrónica), $n^{\circ} 2$. 
Díez Sánchez (Marta) : 1997, « La visita de residencia como instrumento de control de la monarquía sobre el municipio foral : el caso de Alicante », P. Fernández Albaladejo éd., Monarquía, imperio y pueblos en la España moderna. Actas de la IV Reunión Científica de la Asociación Española de Historia Moderna. Alicante, 27-30 de mayo de 1996, Caja de Ahorros del Mediterráneo, Universidad de Alicante, AEHM, 1997, p. 561-568.

Döberl (Mario) : 2007, « La visita generale di Marcos Marañón y Lara nel regno di Sardegna (1714/1715). Un breve periodo di riforme sotto il governo degli Asburgo Austriaci », Estudis, 33, p. 225-253.

Donoso Anes (Alberto) : 1999, « Nuevo método de cuenta y razón para la Real Hacienda en las Indias. La instrucción práctica y provisional en forma de advertencias comentada (27 de abril de 1784) ", Revista española de financiación y contabilidad, n 101, juillet-septembre 1999, p. 817-862.

Dubet (Anne) : 2007, « Les calculs des gens d'argent : des traités d'arithmétique pour marchands et financiers en Espagne au XVIII ${ }^{\mathrm{e}}$ siècle » dans Jean-Pierre Clément, Béatrice Pérez et Sonia V. Rose (coord.), Des marchands entre deux mondes: pratiques et représentations en Espagne et en Amérique (XVe-XVIII ${ }^{e}$ siècles), Paris, Presses de l'Université Paris Sorbonne, p. 323-338.

Dubet (Anne) : 2008a, Un estadista francés en la España de los Borbones. Juan Orry y las primeras reformas de Felipe V (1701-1706), Madrid, Biblioteca Nueva.

Dubet (Anne) : 2008b, « La nueva política crediticia de la corona a principios del siglo XVIII: la creación del Tesorero Mayor de Guerra en España (1703-1706) », Studia Historica. Historia moderna, vol. 30, p. 191-216.

Dubet (Anne) : sous presse, notice « Fraude » dans Marie-Laure Legay et alii, Dictionnaire historique de la comptabilité publique, Genève, Droz, sous presse (2010).

Dubet (Anne) et Negredo del Cerro (Fernando) : 2001, « Le pouvoir de la grâce: le projet politique d'Hernando de Salazar, confesseur du comte-duc d'Olivares » dans Jean-Philippe Luis (dir.), Culture et pouvoir en Espagne (XVI $-\mathrm{XX}^{e}$ siècles). Siècles. Cahiers du Centre d'Histoire « Espaces et Cultures ", $\mathrm{n}^{\circ} 13$, p. 19-28.

Dubet (Anne) éd. : 2008, Les finances royales dans la monarchie espagnole (XVIe-XIXe siècles), Rennes, Presses Universitaires de Rennes,

Elliott (John-Huxtable) : 1990, El Conde-Duque de Olivares. El político en una época de decadencia, Barcelone, Crítica.

Esteban Estríngana (Alicia) : 2002, Guerra y finanzas en los Países Bajos católicos. De Farnesio a Spínola (1592-1633), Madrid, Laberinto.

Esteban Estríngana (Alicia) : 2008, « Le Payeur Général de l'armée des Flandres (1600-1650) : un trésorier incontrôlable? » dans Dubet éd. : 2008, p. 121-136.

Felipo Orts (Amparo) : 1994, « Las visitas de inspección. Un intento de solución de la crisis financiera de la ciudad de Valencia durante el siglo XVII », Estudis, nº 20, 1994, p. 143-165.

Franch Benavent (Ricardo) : 2002, « Las oportunidades de enriquecimiento ilícito generadas por el ejercicio de la intendencia más ‘tentadora'de España: la pesquisa realizada al marqués de Avilés como intendente de Valencia en 1762 ", Estudis, revista de historia moderna, 2002, vol. 28, n 1, p. 263-285

Bernal (Antonio Miguel) : 1992, La financiación de la Carrera de Indias (1492-1824). Dinero y crédito en el comercio colonial español con América, Fundación El Monte, Séville, Consorcio urbanístico del pasillo verde ferroviario de Madrid, Madrid. 
García García (Carmen), 1994, « Oligarquías urbanas y haciendas municipales: irregularidades en la gestión de los fondos locales vallisoletanos en el siglo XVIII », Hacienda Pública Española, I, p. 101-12.

Giménez López (Enrique) et Pradells Nadal (Jesús) : 1989, «Conflictos entre la intendencia y la capitanía general de Valencia durante el reinado de Felipe V. Las denuncias de corrupción », Studia Historica. Historia moderna, vol. VII, p. 591-599.

Gupta (Akhil) : 1995, «Blurred Boundaries: The Discourse of Corruption, the Culture of Politics, and the Imagined State ", American Ethnologist, Vol. 22, No. 2, p. 375-402.

Gutiérrez Nieto (Juan Ignacio) : 1986, «El pensamiento económico, político y social de los arbitristas », dans Historia de España Menéndez Pidal, El siglo del Quijote (1580-1680): Religión, filosofía, ciencia, Madrid, Espasa Calpe, p. 235-354.

Hacienda pública española, ( $\left.\mathrm{n}^{\circ} 1\right), 1994$. Numéro monographique sur la fraude.

Hamon, Philippe : 1999, « Messieurs des finances ». Les grands officiers de finances dans la France de la Renaissance, Paris, CHEFF.

Heras Santos (José Luis de las) : 1991, La justicia penal de los Austrias en la Corona de Castilla, Salamanque, Universidad de Salamanca.

Hernández (Bernardo), 2003, Fiscalismo y finanzas en la Cataluña moderna, Barcelone, Taller de estudios hispánicos e hispanoamericanos.

Hernandez Esteve (Esteban) : 1986, Establecimiento de la partida doble en las cuentas centrales de la Real Hacienda de Castilla, Madrid, Banco de España.

Hespanha (Antonio M.) : 1993, La gracia del derecho. Economía de la cultura en la Edad Moderna, Madrid, Centro de Estudios Constitucionales.

Legay (Marie-Laure) : 2009, « La réforme comptable à Vienne au temps de Marie-Thérèse : Genèse de la nouvelle comptabilité camérale », Histoire et mesure.

Lemarchand (Yannick) : 1997, « Comptabilité et contrôle, une expérience d'introduction de la partie double dans les finances publiques sous la régence ", L'administration des finances sous l'Ancien Régime. Colloque tenu à Bercy les 22 et 23 février 1996, Paris, CHEFF, p. 129-154.

Madrazo (Santos) : 2000, Estado débil y ladrones poderosos en la España del siglo XVIII. Historia de un peculado en el reinado de Felipe $V$, Madrid, Catarata.

Mény (Yves) : 1992, La corruption de la république, Paris, Fayard, 1992.

Molas Ribalta (Pere) : 1988, « Sàtira política a Valencia el 1735 », Homenatge al doctor Sebastià García Martínez, vol. II, Valence, Conselleria de Cultura, p. 313-326.

Moreno Cebrián (Alfredo) et Sala i Vila (Núria) : 2004, El « premio » de ser virrey. Los intereses públicos y privados del gobierno virreinal en el Perú de Felipe V, Madrid, CSIC.

Nava Rodríguez (Teresa) : 2004, «'Nervios de bóveda’: las tesorerías centrales de la hacienda borbónica (1716-1743)» dans Martínez Ruiz(Enrique) coord., III seminario hispanovenezolano. Vínculos y sociabilidades en España et Iberoamérica. Siglos XVI-XX. Ciudad Real, p. 111-113.

Olivier de Sardan (J.P.) : 1999, « A Moral Economy of Corruption in Africa? », The Journal of Modern African Studies, Vol. 37, No. 1, p. 25-52.

Patisso (Giuseppe) : 2009, Visitas de Italia". Un caso di corruzione nella Puglia del Seicento: "il proceso contra Muzio De Angelis, perceptor de la provincia de Bari. 
Peytavin (Mireille) : 2003, Visite et gouvernement dans le royaume de Naples (XVI ${ }^{e}$-XVII ${ }^{e}$ siècles), Madrid, Casa de Velázquez.

Planas (Natividad) : 2000, Pratiques de pouvoir au sein d'une société frontalière. Le voisinage $\mathrm{du}$ Royaume de Majorque et ses îles adjacentes avec les terres d'Islam au XVII siècle, Thèse de doctorat inédite, Institut Européen de Florence.

Pro Ruiz (Juan) : 2008, « Des finances royales aux finances publiques : le contribuable et l'administration dans le nouvel ordre fiscal (1833-1852) » dans Dubet éd. : 2008, chap. 19, p. 291-305.

Rowlands (Guy) : 2002, The Dynastic State and the Army under Louis XIV. Royal Service and Private Interest, 1661 to 1701, Cambridge, Cambridge University Press.

Ruiz Martín (Felipe) :1968, «Las finanzas españolas durante el reinado de Felipe II », Cuadernos de historia, Anexos de la revista Hispania, $\mathrm{n}^{\circ}$ 2, p. 109-173.

Sabatini (Gaetano) : 1997, Il controllo fiscale sul territorio nel Mezzogiorno spagnolo e il caso delle province abruzzesi, Naples, Istituto Italiano per gli Studi filosofici.

Sánchez Belén (Juan A.), 1996, La política fiscal en Castilla durante el reinado de Carlos II, Madrid, Siglo XXI.

Waquet (Jean-Claude) : 1984, De la corruption. Morale et pouvoir à Florence aux XVII et XVIIIe siècles, Paris.

\section{ANNEXES}

\section{Abréviations}

AGS : Archives Générales de Simancas

SSH: Secretaría y Superintendencia de Hacienda

AHN : Archivo Histórico Nacional

E : Estado

\section{Sources documentaires}

Barrème, Traité des parties doubles ou Méthode aisée pour apprendre à tenir en Parties Doubles les Livres du Commerce \& des Finances, avec un Traité de Finance, Paris, 1721.

Bielfeld, baron de, Institutions politiques, par (...),Tome Premier, A La Haye, chez Pierre Gosse Junior, Libraire de SAS Monseigneur le Prince Stathouder, 1760.

Centurión (Juan) : 1593, Oposiciones que hizo contra los Erarios don Juan Centurión, Caballero Genovés, Marqués de Estepa, dans Luis Valle de la Cerda, Desempeño del patrimonio de Su Majestad y de los Reinos, sin daño del Rey y vasallos, y con descanso y alivio de todos, por medio de los Erarios Públicos y Montes de Piedad, Madrid, Pedro Madrigal, 1600.

Déclaration concernant la tenue des registres journaux, Versailles, 4 octobre 1723. 
Dictionnaire des finances, contenant la définition de tous les termes de Finance, leur usage et leurs différentes applications dans toutes sortes d'affaires. L'explication de tous les droits et impositions qui se lèvent dans l'étendue du royaume. L'établissement des Conseils du roi, des Chambres des Comptes et des Cours des Aides, la création des Charges, et généralement tout ce qui regarde les Finances et la pratique des Bureaux. A Paris, Jacques Josse, Théodore Le Gras, Guillaume Cavelier, La Veuve Saugrain, 1727, Avec approbation et privilège du roi.

Édit concernant les registres-journaux qui doivent être tenus par tous les officiers comptables \& autres chargés de la perception, maniement \& distribution des finances du Roy, \& des deniers publics, du mois de juin 1716.

Pâris la Montagne, Traité des administrations des recettes et dépenses du Royaume, 1733

(A.N. KK 1005 C).

Ripia, Juan de la, Práctica de la administración y cobranza de las rentas reales y visita de los ministros que se ocupan en ellas, Madrid, 1676.

Alonso García (David) : 2007a, Fisco, poder y monarquía en los albores de la modernidad Castilla, 1504-1525. Thèse de doctorat téléchargeable sur http://eprints.ucm.es/tesis/ ghi/ucm-t27728.pdf

\section{NOTES}

1. Pour un bilan historiographique, voir l'introduction de Dubet: 2008a. (NB : les références bibliographiques complètes sont indiquées à la fin de l'article). Une bibliographie complète : voir Dubet éd. : 2008.

2. Pro Ruiz : 2008.

3. Numéro monographique de Hacienda pública española, 1994; Cárceles de Gea: 1995, et 2000 ; Pro Ruiz : 2008.

4. Bernardo Ares : 1993 ; Felipo Orts : 1994 ; Díez Sánchez : 1997 ; Alonso García : 2008 ; Carmen García García : 1994 ; Sánchez Belén : 1996 ; Hernández : 2003.

5. Ruiz Martín : 1968 ; Alonso García : 2007 aet 2007 b.

6. Molas Ribalta : 1988 ; Giménez López et Pradells Nadals : 1989 ; Bertrand : 1999.

7. Peytavin: 2003; Esteban Estríngana: 2002 ; Bertrand: 2008; Dubet: sous presse. Les questionnaires adressés aux témoins au cours des visites (enquêtes) dont font l'objet les administrateurs des recettes royales sont autant de descriptions de ces délits (Ripia: 1676, p. 379-387).

8. Moreno Cebrián et Sala i Vila : 2004.

9. Cuartas Rivero : 1984 ; Sánchez Belén : 1996 ; Bertrand : 1999 ; Peytavin : 2003.

10. Par exemple, avec les fraudeurs pour le prélèvement des taxes sur le commerce avec l'Amérique ou la Méditerranée ou les produits objets de monopoles (Elliott : 1991 ; Bernal : 1992 ; Planas : 2000) ou avec des partisans qui sont tous un tant soit peu usuriers (Ruiz Martín : 1968). En 1714, le visiteur général de Sardaigne préfère infliger un châtiment exemplaire au plus coupable des officiers du Patrimoine, plutôt que de mener une enquête longue, qui menacerait le fonctionnement de l'administration, sur les agissements de ses collègues et complices (Döberl : 2007, p. 250).

11. Un des expédients prisés par les donneurs d'avis dont le Conseil des Finances archive soigneusement les propositions consiste à entamer des poursuites contre telle ou telle catégorie d'employés des finances pour en tirer des amendes. Gutiérrez Nieto : 1986 . 
12. Waquet : 1984 ; Sabatini : 1997 ; Hamon : 1999 ; Rowlands : 2002 ; Dessert : 2004 ; Peytavin : 2003 ; Döberl : 2007 ; Patisso : 2009.

13. Waquet: 1984.

14. Gupta : 1995 ; Olivier de Sardan : 1999.

15. Plusieurs sociologues, économistes et anthropologues affirment qu'elle n'est pas un résidu de l'Ancien Régime, mais fait partie du fonctionnement de certaines démocraties. Voir l'état de la question dressé par Mény : 1992. Pour l'Afrique contemporaine : Olivier de Sardan : 1999.

16. Waquet: :1984, p. 29.

17. Waquet :1984, p. 61-64 et 134-136.

18. Waquet :1984, p. 149-177.

19. Madrazo : 2000, p. 53.

20. Madrazo : 2000, p. 64 .

21. Madrazo : 2000, p. 92-95.

22. Madrazo : 2000, conclusion.

23. Madrazo : 2000, p. 47-52, 54-48, 164-170.

24. Peytavin 2003, passim. Des exemples comparables : Díaz Ordoñez : 2003 ; Franch Benavent : 2002.

25. Dubet et Negredo del Cerro : 2001

26. Clavero : 1991.

27. Heras Santos : 1991, passim ; Clavero : 1996.

28. Clavero : 1986 ; Hespanha : 1993, passim.

29. Waquet: 1984 , p. 150-158.

30. Plusieurs exemples dans Esteban Estríngana : 2002, passim et Carlos Morales, 2000, passim. Voir aussi Ripia : 1676, p. 219-220, 379-387. C'est pour mettre fin à l'omission en recette et au faux emploi en dépense que la tenue de registres journaux est imposée aux comptables en France en 1716 et 1723 (Édit concernant les registres-journaux qui doivent être tenus par tous les officiers comptables \& autres chargés de la perception, maniement \& distribution des finances du Roy, \& des deniers publics, juin 1716 ; Déclaration concernant la tenue des registres journaux, Versailles, 4 octobre 1723).

31. Sur la comptabilité en recette, dépense et reprise : Esteban Estríngana : 2008 ; Legay : 2009.

32. Sur l'intérêt d'introduire la partie double dans les finances publiques, Barrème : 1721, passim ; Pâris la Montagne : 1733 (je dois cette référence à Yannick Lemarchand, que je remercie). Des tentatives d'introduction de la partie double dans les finances de la France et de la monarchie espagnole, entre le XVIe et le XVIIIe siècle, se soldent par des abandons (Lemarchand: 1997 ; Donoso Anes : 1999 ; Hernández Esteve : 1986). A la fin du XVIIIe siècle, le baron de Bielfeld, qui a affirmé dans ses Institutions politiques que la partie double ne peut convenir aux finances d'un prince (1760, chap. 12, § 35), est une référence (Donoso Anes : 1999).

33. C'est le cas de Bartolomé Spínola vers 1632 (Andrés Ucendo : 1999, p. 59). «Si les receveurs présentent des cautions - écrit le Génois Juan Centurión en 1593 -, c'est en raison de l'utilité qu'ils tirent de l'argent, en retardant les paiements. Et c'est avec ce gain qu'ils secourent leurs cautions, c'est bien connu, et parfois même leur paient leurs garanties. » (Centurión : 1593, fol. 77). Toutes les sources citées sont à l'origine en espagnol : c'est moi qui traduis.

34. AGS, SSH, leg. 213, 214 et 215 ; AHN, E, leg. 768 et 2974. Le dossier pourrait être incomplet. En décembre 1737, Verdes Montenegro prie le roi de le transférer en un «lieu public ", car une partie a peut-être déjà brûlé dans l'incendie du Palais Royal, le reste étant chez le secrétaire de la junte qui a instruit l'affaire. En mars 1738 le dossier est versé aux archives de la " voie réservée » (AHN, E, leg. 2974).

35. AGS, SSH, leg. 210 et 213 ; AHN, E, leg. 768.

36. Sur cette chronologie, Nava Rodríguez : 2004 ; Dubet : $2008 \mathrm{~b}$.

37. Sur ces créations : Dedieu : 2000 ; Nava Rodríguez : 2004 ; Castro : 2004 ; Dubet : 2008 a.

38. Madrazo : 2000, p. 68-95. 
39. Castro : 2004 , p. $360-361$

40. Miraval à don Juan Bautista de Orendain, 02/02/1724, Madrid, AHN, E, leg. 768. Miraval précise que Verdes assume de fait la fonction en raison de la maladie de Campoflorido et critique ce dernier pour « la distribution volontaire qu'il faisait de quelques paiements effectués pour des dettes qui n'étaient pas aussi urgentes que d'autres, qui demandaient satisfaction plus rapide ».

41. AHN, E, leg. 768 et leg. 884 ; AGS, SSH, leg. 213.

42. AHN, E, leg. 768. Les récits donnés par la défense de Verdes Montenegro relatent l'incident (AHN, E, leg. 2974).

43. Il s'en plaint à José Grimaldo, Secrétaire des Dépêches et d'État et homme de confiance de Philippe V, le 23/09/1724 : « depuis que j'ai mérité l'honneur d'occuper l'emploi de Secrétaire, la haine de quelques-uns s'est conjurée contre moi, sans aucun doute parce que je fais obstacle à leurs projets » (AGS, SSH, leg. 214).

44. Castro : 2004, p. 374-379.

45. «Extracto ajustado de los papeles de la causa de don Fernando Verdes Montenegro, que se formó para hacer relación en las juntas que conocieron de ella y informar a SM », AHN, E, leg. 2974.

46. "Extracto ajustado », AHN, E, leg. 2974, fol. 19.

47. Don Joseph de Castro, don Francisco de Arriaza, don Jacinto de Arana, don Joseph Llopiz et don Lorenzo de Medina au roi, 15/06/1727. Le secrétaire de la junte est don Antonio López Salces. AHN, E, leg. 2974

48. "La junta mandada formar por VM », don Joseph de Castro y Araujo, don Rodrigo de Cepeda, don Baltasar de Acevedo, don Gonzalo Machado, don Francisco de Arriaza à Philippe V, 26/10/1724, AHN, E, leg. 2974.

49. Voir Ibidem.

50. La liste de ces complices a été remise à la junte qui s'est prononcée le 26/10/1724 (« Noticias concernientes a la causa de D. Fernando Verdes Montenegro », AHN, E, leg. 2974).

51. Lettres à Orendain, 12/07/1725 et 06/09/1725, AGS, SSH, leg. 215.

52. «Extracto ajustado», AHN, E, leg. 2974, fol. 10.

53. Ibidem.

54. Hinojosa à José Grimaldo, Madrid, 15/09/1724, AGS, SSH, leg. 214-2. "Extracto ajustado ", AHN, E, leg. 2974, fol. 10.

55. Ibidem.

56. Ibid.

57. " Extracto ajustado ", AHN, E, leg. 2974, fol. 33.

58. Lettre à José Grimaldo, Madrid, 23/09/1724, AGS, SSH, leg. 214-2.

59. AHN, E, leg. 768.

60. Lettre à Grimaldo, Madrid, 23/09/1724, AGS, SSH, leg. 214-2.

61. Lettre à Grimaldo, Madrid, 26/09/1724, AGS, SSH, leg. 214-2.

62. Lettre à Grimaldo, Madrid, 10/10/1724, AGS, SSH, leg. 214-2.

63. Miraval à Orendain, Madrid, 02/02/1724, AHN, E, leg. 768.

64. « Extracto ajustado », AHN, E, leg. 2974, fol. 33.

65. Verdes Montenegro à Louis Ier, 06/06/1724, lettre rapportée dans AHN, E, leg. 2974, fol. 3. Le même à Grimaldo, Madrid, 13/09/1724, AGS, SSH, leg. 214-2.

66. Défense de Verdes Montenegro, dans le compte-rendu de la junte du 15/06/1727, AHN, E, leg. 2974.

67. «Extracto ajustado ", AHN, E, leg. 2974, fol. 12.

68. Ibidem, fol. 23.

69. Ibid.

70. Bien sûr, cela ne résout en rien la question de savoir par quel processus les choses changent entre le début du XVIII ${ }^{e}$ siècle et le XIXe siècle. 
71. Elle se traduit par la publication de livres mettant en avant ce savoir, qui n'est ni celui des banquiers ou des marchands, ni celui des juges, ni celui des arithméticiens. Dubet : 2007.

\section{AUTEUR}

\section{ANNE DUBET}

Université Blaise Pascal Clermont-Ferrand, Centre d'Histoire « Espaces et Cultures »* 